\title{
Impact ionization coefficient ratio in InGaAs/InAlAs superlatice avalanche photodiodes determined from noise measurements
}

\author{
Young-June Yu and Gils Bosman \\ Department of Electrical Engineering, University of Florida, Goinesville, Florida 32611 \\ P. K. Bhattacharya \\ Deparment of Electrical Engineering and Computer Seience, University of Michigan, Ann Arbor, \\ Michigan 48709
}

(Received 22 Jure 1987; accepted for publication 8 September 1987)

\begin{abstract}
The noise of an $I n_{0.53} G a_{0.47} \mathrm{~A} \$ / \operatorname{In}_{0.52} \mathrm{Al} l_{0.48} \mathrm{As}$ superlatice avalanche photodiode is measured at $700 \mathrm{MHz}$ as a function of applied reverse bias voltage. From the measured data the ratio $\mathrm{k}$ of the hole to electron impact ionization coeffeients is determined. This ratio is equal to 6 in the field range $(0.8-2.3) \times 10^{5} \mathrm{~V} / \mathrm{cm}$; beyond this range $k$ decreases $w i n$ increasing feld. The field dependence of $k$ is atiributed to a transition from ionization across the valerce-band-edge discontinuity to band-to-band lonization.
\end{abstract}

Recently, renewed interest has been given to $p^{+}+i n n^{+}$ avalanche photodiodes (APD's) because superlatice $i$ regions are able to enhance the difference between the impact ionization coeficients $\alpha$ and $\beta$ for electrons and holes, which is an essential requirement for low-noise photodetection.

Since the superlatice APD structures were first proposed, experimental evidence of enhancenent of the ionization coeficient ratio has been reported with values of $\alpha / \beta$ up to 8 for GaAs/AIGaAs APD's. ${ }^{2,3}$ This enhancement of the ionization coeficient ratio is attributed to the fact that the conduction-band discontinuity is larger than the valenceband discontinuiry, ${ }^{2}$ and as a result electrons gain more $\mathrm{ki}$. netic energy in traversing a heterojunction interface than holes do. Since this sudden gain in kinetic energy increases the probability for impact ionization, $\alpha$ becomes larger than B. More recently, Capasso et al. ${ }^{4}$ fabricated $\mathrm{In}_{0.53} \mathrm{Ga}_{0.47} \mathrm{AS}$ $\mathrm{In}_{0.52} \mathrm{Al}_{0.48} \mathrm{As}$ superlattice APD's and reported that $\alpha / \beta \simeq 0.05$. This result is surprising since it cannot be explained with the model discussed above. Capasso et al pointed out, however, that for this structure and for the fieid range considered, the impact ionization of hot carriers with carriers thermally generated and dynamically stored in the quancum wells is responsible for the low feld multiolicetion. This mechanism was first discussed by Smith et al. and later by Chuang and Hess." Since the valence-band discontinuity $\triangle E_{\nu}$ is much less than the conduction-band discontinuity $\Delta E_{c}$ at an $\mathrm{In}_{0.52} \mathrm{Ga}_{0.47} \mathrm{As} / \mathrm{In}_{0.52} \mathrm{Al} \mathrm{l}_{0.48} \mathrm{As}$ interface, the hole confinement is weaker than the clectron confinement. As a consequence the holes get released more easily than the electrons from the quantum wells. This mechanism is referred to as "ionization across the band-edge discontinuity."

In our study we used an undoped $\ln _{0.53} \mathrm{Ga}_{0.47} \mathrm{As}(90$ A) $/ \operatorname{mos}_{0.52} \mathrm{Al}_{0.48} \mathrm{As}(90 \AA)$ superlattice structure. The impact ionization coeficient ratio presented in this letter was determined from noise experiments for electric field strengths ranging from $0.8 \times 10^{5}$ to $2.7 \times 10^{5} \mathrm{~V} / \mathrm{cm}$. The experimental data indicate that for a fraction of this range $(0.8-2.3) \times 10^{5} \mathrm{~V} / \mathrm{cm}$, impact ionization across the valence. band-edge discontinuity is responsible for the avalanche multiplication.

As shown in the insert of Fig. 1, our devices are mesa


lattice matched to an $n^{+}$-InE substrate was grown, followed by 1.8 - $4 \mathrm{~m}$-thick undoped $\ln _{0.53} \mathrm{Ga}_{0.47} \mathrm{As} / \mathrm{In}_{0.52} \mathrm{Al} \mathrm{H}_{3.48} \mathrm{As}$ superlattice region consisting of 100 alternating layers and a 2-km-thick $p^{+}-\mathrm{In}_{0.53} \mathrm{Ga} \mathrm{a}_{0.47}$ As layer for the photoabsorption. For these mole fractions the band-edge discontinuities at the heterojunction interfaces are $0.5 \mathrm{eV}$ for the conduction-band edge and $0.2 \mathrm{eV}$ for the valence-band edge. ${ }^{7}$ The samples were grown using molecular beam epiaxy (MBE). The top layer of the $p^{+}-i-n^{+}$diodes is iluminated by a 1.3 . $\mu \mathrm{m} \operatorname{lnGaA}$ light-emitting diode. An optical n̂ber was used to guide and focus the beam on the APD's. The narrow gap $\left(E_{g}=0.75 \mathrm{eV}\right)$ InGaAs material absorbs most of the incoming light, resulting in pure electron injection into the high feld superiatice region where multiplication takes place.

Dark current and photocurrent measurements with continuous illumination were performed as a function of reverse bias voltage, and the results are shown in Fig. 1. The

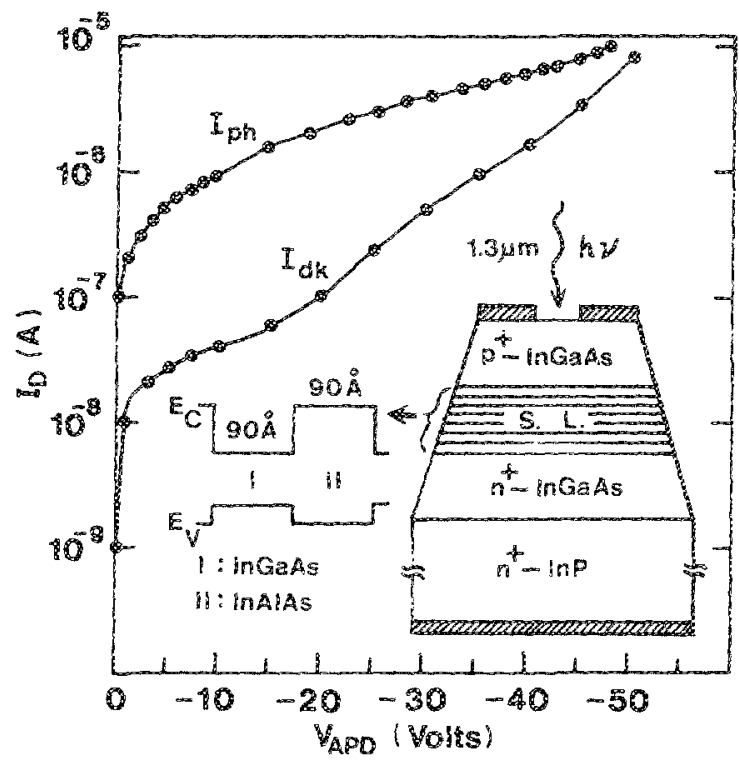

FIG. 1. Dark current and photow urent characteristics. The insert shows the device configuration and the band diagram of the superlatice structure. 
rapid increase in the photocurrent between 0 and $10 \mathrm{~V}$ is due to the enhanced collection of photogenerated carriers across the superlattice region with increasing electric field. ${ }^{8}$ Above $15 \mathrm{~V}$ the dark current increase is in part due to impact ionization and in part due to the Zener efiect taking place in the quantum wells. The device temperature was $300 \mathrm{~K}$ during our experiment.

Noise measurements performed between $10 \mathrm{~Hz}$ and 25 $\mathrm{kHz}$ at room temperature revealed large excess noise levels, probably associated with trapping and detrapping of carriers in quantum wells or traps. These large noise levels result in corner frequencies well above $1 \mathrm{MHz}$, and a high-frequency, low-noise receiver system had to be designed to measure the noise associated with avalanche multiplication. We conbined the advantages of Gasquet's circulator method" and Dicke's modulation technique ${ }^{10}$ to build a receiver with a detection limit of $10^{-6}$ A equivalent noise current and a bandwidth ranging from 500 to $1000 \mathrm{MHz}$.

Figure 2 shows our experimental setup. The device is mounted on a 50- $\Omega$ microstrip line with a 50- $\Omega$ termination. By slowly $(0.2 \mathrm{~Hz})$ switching between port 1 and port 2 of the microwave switch, the current noise generated in the $A P D$ is compared with the reference noise source and averaged for a long time interval. In this way the weak current noise signal which is much smaller than the thermal noise generated in the 50- $\Omega$ load resistor can be extracted from the background noise.

In Fig. 3 we present the current noise spectral density measured at $700 \mathrm{MHz}$ versus illuminated diode current. Measurements at other frequencies between 500 and 1000 $\mathrm{MHz}$ gave similar results, indicating that the noise is independent of frequency.

A common way to express the current noise $S_{i}$ of an $\mathrm{APD}$ is

$$
S_{i}=2 e I_{p x} M^{2} F
$$

where $M$ is the multiplication factor defined by the ratio of output current over the primary current and $F$ is called the excess noise factor. The primary current $I_{p r}$ is determined from the breakpoint in Fig. 3 where the slope of $S_{i}$ vs $I_{B}$ changes abruptly due to the onset of avalanche multiplication. From Fig. 3 we find $I_{\mathrm{pr}}=1.6 \mu \mathrm{A}$.

In terms of Mclntyre's theory ${ }^{11}$ for band-to-band ionization, $F$ is given by

$$
F=M\left\{1-(1-k)[(M-1) / M]^{2}\right\}
$$

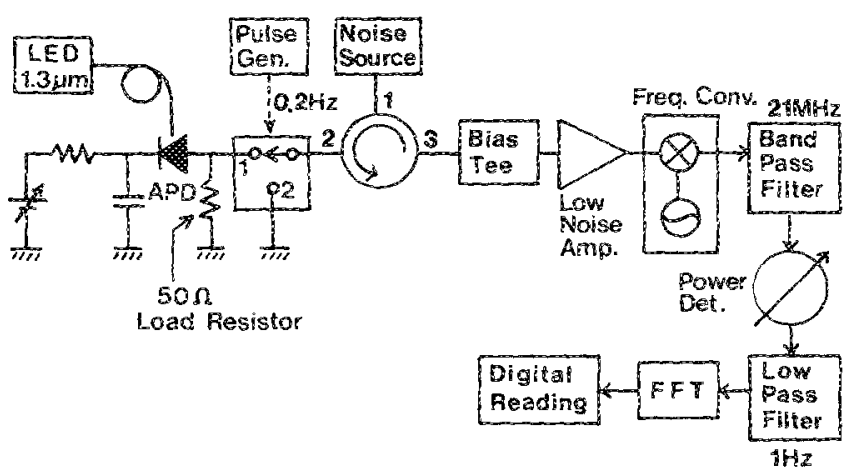

FIG. 2. High-frequency noise measurement setup.

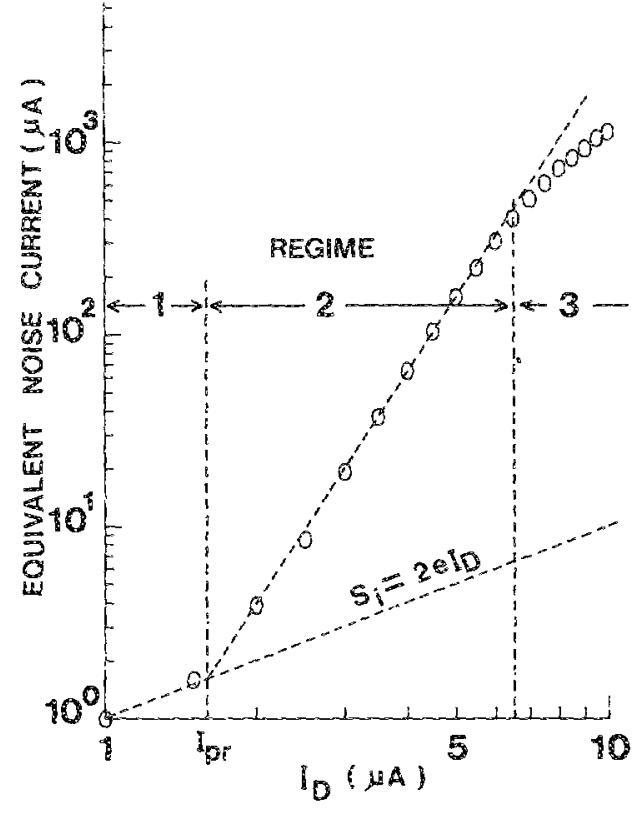

FIG. 3. Measured current noise spectral density vs diode current. Regimes 1,2 , and 3 represent different modes of device operation.

for the case when the primary carriers injected into the avalanche region are electrons. The factor $k$ is equal to $\beta / \alpha$. We assume that Eq. (2) also predicts the excess noise caused by ionization across the band-edge discontinuity. In our device, for example, a bole-initiated band-edge ionization triggers the release of a dynamically stored electron from the corresponding quantum well in the conduction band. This release of an electron is required to preserve current continuity. Therefore, ionization across the band-edge discontinuity can be effectively described as a three-particle, band-to-band ionization process and Mclntyre's formalism should apply.

In Fig. 4 the experimental values of $F$ as calculated from Eq. (1) are plotted versus the multiplication factor $M$. Also included are theoretical curves for $F$ calculated using Eq. (2). Note that a breakpoint occurs at $I_{D}=6.5 \mu \mathrm{A}$ or $V_{A P D}=41 \mathrm{~V}$, which corresponds to an electric fieid of $2.3 \times 10^{5} \mathrm{~V} / \mathrm{cm}$. Beyond this point the curve of $F$ vs $A$ be-

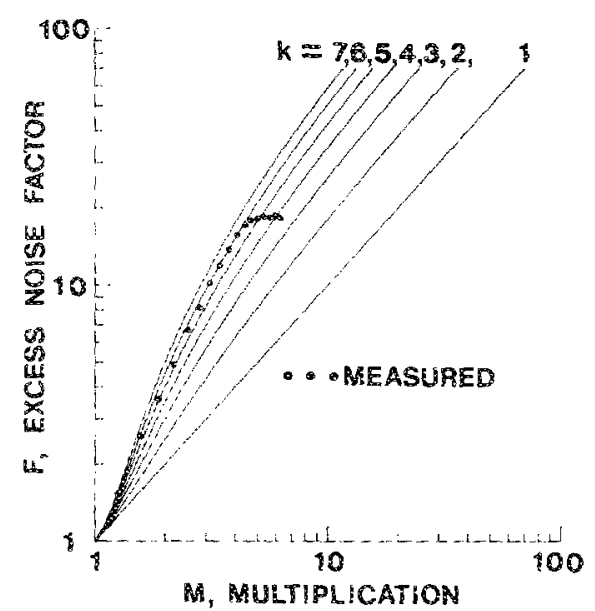

FIG. 4. Excess noise factor vs multiplication. The solid lines indicate theoretical curves for different values of $k .^{11}$ 
comes fiat and approaches the curve for $k=1$. This means that the difference between $\beta$ and $\alpha$ becomes smaller with increasing bias. To explain these results, we introduce three different regimes of operation for this superlattice $A \mathrm{PD}$ (see Fig. 3).

Regine $7: 0<V<15 \mathrm{~V}$. No multiplication occurs and the noise is equal to full shot noise.

Regime 2: $15 \mathrm{~V}<V<41 \mathrm{~V}$. The onset electric field of avalanche multiplication is $8 \times 10^{4} \mathrm{~V} / \mathrm{cm}$, which is in good agreement with the value reported by Capasso. This multplication at low field strengths and the enhancement of $\beta$ over $\alpha$ are strong indications that impact ionization across the band-edge discontinuity is taking place. Ionization across the band-edge discontinuity is dominant in the valence band because the valence-band discontinuity $\left(\Delta E_{v}=0.2 \mathrm{eV}\right)$ is smaller than the conduction-band discontinuity $\left(\Delta E_{c}=0.5 \mathrm{eV}\right)^{7}$; consequently, $\beta$ is larger than $\alpha$, since it is easier to ionize holes than electrons from the quantum wels.

Regime 3: $41 \mathrm{~V}<V$. Band-to-band ionization sets in, making $\alpha$ and $\beta$ roughly equal and thereby reducing the value of $k$. In addition, at these high bias levels the leakage current becomes quite large, mainly due to the Zener effect. This resuits in mixed current injection into the avalanche region and limits the validity of our discussion, which as- sumes pure electron injection. Note that the high field breakpoint in the $F$ vs $M$ curve occurs at $2.3 \times 10^{5} \mathrm{~V} / \mathrm{cm}$, which is very ciose to the value of $2.5 \times 10^{5} \mathrm{~V} / \mathrm{cm}$ Capasso et al. obtained $^{12}$ for the onset of band-to-band ionization.

This study was supported by Micrafabritech at the University of Florida.

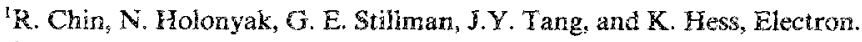
Letr. 16, $467(1980)$.

${ }^{2}$ F. Capesso, W. T. Tsang, A. L. Hutchinson, and G. F. Wilhiums, Appl. Phys. Lett. 40, 38 (1982).

${ }^{3}$ F. Y. Juang, U. Das, Y. Nashimoto, and P. K. Bhattacharya, Appl. Phys. Lett. $47,972(1985)$.

${ }^{4}$ E. Capasso, J. Allam, A. Y. Cho, K. Mohammed, R. J. Malik, A. L. Huichinson, and D. Sivco, Appl. Plyys. Let1. 48, 1294 (1986).

5. S. Smith, L. C. Chiu, S. Margalit, A. Yariv, and A. Y. Cho, J. Vac. Sei. Technol. B 1, 376 (1983).

5. L. Chuang and K. Hess, J. Appi. Phys. 59, 2885 (1986).

${ }^{7}$ R. People, K. W. Wecht, K. Alaw, and A. Y. Cho, Anpl. Phys. Lett. 43, 188 (1983).

${ }^{8}$ J. Allam, F. Capasso, K. Alavi, and A. Y. Cho, IEEE Electron Device Lett. EDL-8, 4 (1987).

'D. Gasquet, J. C. Vassiere, and J. P. Nougier, Sixth International Conference on Notse in Physical Systems (NBS Special Publication 614, 1981), p. 305.

${ }^{10}$ R. H. Dicke, Rev. Sci. Instrum. 17, 268 (1946).

i'R. J. Mchntyre, IEEE Trans. Electron Devices ED-13, 164 (1966).

"K. Mohammed, F. Capasso, J. Allam, A. Y. Cho, and A. L. Hutchinson, Appl. Phys. Lett. 47, 597 (1985). 